\title{
A Lean Tactic for Normalising Ring Expressions with Exponents (Short Paper)
}

\author{
Anne Baanen ${ }^{(凶)}(\mathbb{D}$ \\ Vrije Universiteit Amsterdam, Amsterdam, The Netherlands \\ t. baanen@vu.nl
}

\begin{abstract}
This paper describes the design of the normalising tactic ring_exp for the Lean prover. This tactic improves on existing tactics by extending commutative rings with a binary exponent operator. An inductive family of types represents the normal form, enforcing various invariants. The design can also be extended with more operators.
\end{abstract}

\section{Introduction}

In interactive theorem proving, normalising tactics are powerful tools to prove equalities. Given an expression $a$, these tactics return an expression $a^{\prime}$ in normal form together with a proof that $a=a^{\prime}$. For instance, in mathlib [10], the mathematical library for the Lean theorem prover [7], the ring tactic normalises expressions in a commutative (semi)ring. Analogous tactics or conversions exist in many theorem provers $[5,8,9]$. The ring tactic in Lean can be directly invoked by the user and is called by the decision procedure linarith. The utility of ring is evident from the fact that it is invoked over 300 times in mathlib.

The ring tactic in Lean, and the tactic in Coq it is based on, use a Horner normal form representation of polynomials [4]. The Horner form represents a polynomial $f(x)$ with one of two cases: either it is constant $(f(x)=c)$ or it is of the form $f(x)=c+x * g(x)$. This representation allows ring to uniquely and efficiently represent any polynomial, i.e. any expression consisting of the operators + and $*$, numerals and variables. Problems arise when expressions include other operators than + and $*$, such as the exponentiation operator $\wedge$. The Horner form fundamentally assumes the degree of a term is a constant integer, so it cannot be simply modified to represent variable exponents, or more generally to represent ${ }^{\wedge}$ applied to compound expressions. The analogous procedures in other theorem provers have the same restriction. Adding rewrite rules such as $x^{n+1} \mapsto x * x^{n}$ is not a universal solution. This rule would unfold the expression $x^{100}$ into a large term composed of repeated multiplications, reducing the performance of the procedure significantly. The result is that ring cannot prove that $2^{n+1}-1=2 * 2^{n}-1$ for a free variable $n: \mathbb{N}$.

The ring_exp tactic uses a new extensible normal form, currently supporting the operators,$+ *$ and ${ }^{\wedge}$, numerals and variables. Its domain is a strict superset 
of the domain of previous semiring tactics, without sacrificing too much of the efficiency of ring. This paper describes the design and engineering challenges encountered in implementing ring_exp.

The version of ring_exp discussed in this paper was merged into mathlib in commit 5 c09372658. ${ }^{1}$ Additional code and setup instructions are available online. $^{2}$

\section{Design Overview}

The ring_exp tactic uses a normalisation scheme similar to the original ring tactic. The input from the tactic system is an abstract syntax tree representing the expression to normalise. An eval function maps inputs to a type ex of normalised expressions. The normal form should be designed in such a way that values of type ex are equal if and only if the input expressions can be proved equal using the axioms of commutative semirings. From the ex representation, the normalised output expression is constructed by a function simple. Both eval and simple additionally return a proof showing that the input and output expressions are equal.

The ring_exp tactic does not use reflection but directly constructs proof terms to be type checked by Lean's kernel, as is typical for tactics in mathlib [10]. Reflective tactics avoid the construction and checking of a large proof term by performing most computation during proof checking, running a verified program [2]. If the proof checker performs efficient reduction, this results in a significant speed-up of the tactic, at the same time as providing more correctness guarantees. Unfortunately, the advantages of reflection do not translate directly to Lean. Tactic execution in Lean occurs within a fast interpreter, while the kernel used in proof checking is designed for simplicity instead of efficient reduction [3]. Achieving an acceptable speed for ring_exp requires other approaches to the benefits that reflection brings automatically.

The language of semirings implemented by ring, with binary operators,$+ *$ and optionally - and /, is extended in ring_exp with a binary exponentiation operator ${ }^{\wedge}$. The input expression can consist of these operators applied to other expressions, with two base cases: natural numerals such as 0 and 37, and atoms. An atom is any expression which is not of the above form, e.g. a variable name $x$ or a function application $\sin (y-z)$. It is treated as an opaque variable in the expression. Two such expressions are considered equal if in every commutative semiring they evaluate to equal values, for any assignment to the atoms.

Using a suitable representation of the normal form is crucial to easily guarantee correctness of the normaliser. Since there is no clear way to generalise the Horner form, ring_exp instead represents its normal form ex as a tree with operators at the nodes and atoms at the leaves. Certain classes of nonnormalised expressions are prohibited by restricting which sub-node can occur for each node. The ex type captures these restrictions through a parameter in

\footnotetext{
${ }^{1}$ https://github.com/leanprover-community/mathlib/tree/5c09372658.

${ }^{2}$ https://github.com/lean-forward/ring_exp.
} 


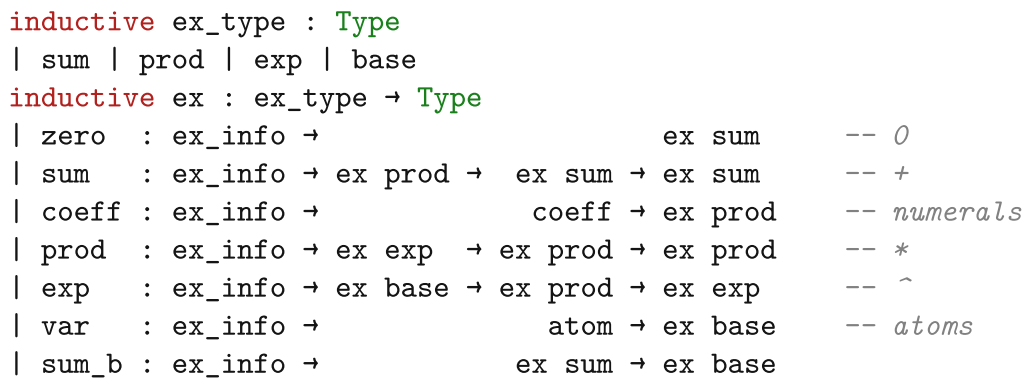

Fig. 1. Definition of ex_type and ex

the enum ex_type, creating an inductive family of types. Each constructor allows specific members of the ex family in its arguments and returns a specific type of ex. The full definition is given in Fig. 1. The additional ex_info record passed to the constructors contains auxiliary information used to construct correctness proofs. The sum_b constructor allows sums as the base of a power, analogously to the parentheses in $(a+b)^{c}$.

For readability, we will write the ex representation in symbols instead of the constructors of ex. Thus, the term sum (prod (exp (var n) (coeff 1)) (coeff 1)) zero (with ex_info fields omitted) is written as $n^{1} * 1+0$, and the normalised form of $2^{n}-1$ is written $(2+0)^{n^{1} * 1} * 1+(-1)+0$.

Table 1. Associativity and distributivity properties of the,$+ *$ and ${ }^{\wedge}$ operators

\begin{tabular}{c|ccc}
\hline & + & $*$ & $\wedge$ \\
\hline+ & $(a+b)+c=a+(b+c)$ & - & - \\
$*$ & $(a+b) * c=a * c+b * c ;$ & $(a * b) * c=a *(b * c)$ & - \\
$\wedge$ & $a *(b+c)=a * b+a * c$ & $(a * b)^{c}=a^{c} * b^{c}$ & $\left(a^{b}\right)^{c}=a^{b * c}$ \\
\hline
\end{tabular}

The types of the arguments to each constructor are determined by the associativity and distributivity properties of the operators involved, summarised in Table1. Since addition does not distribute over either other operator (as seen from the empty entries on the + row), an expression with a sum as outermost operator cannot be rewritten so that another operator is outermost. Thus, the set of all expressions should be represented by ex sum. Since $*$ distributes over

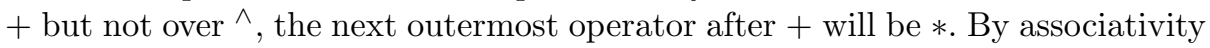
(the diagonal entries of the table) the left argument to + should have $*$ as outermost operator; otherwise we can apply the rewrite rule $(a+b)+c \mapsto a+(b+c)$. Analogously, the left argument to the prod constructor is not an ex prod but an ex exp, and the left argument to exp is an ex base. 
The eval function interprets each operator in the input expression as a corresponding operation on ex, building a normal form for the whole expression out of normalised subexpressions. The operations on ex build the correctness proof of normalisation out of the proofs for subexpressions using a correctness lemma: for example, the lemma add_pf_z_sum $: p s=0 \rightarrow q s=q s^{\prime} \rightarrow^{\prime} p s+q s=q s^{\prime}$ is used on the input expression ps + qs when ps normalises to 0 .

Adding support for a new operator would take relatively little work: after extending the table of associativity and distributivity relations, one can insert the constructor in ex using the table to determine the relevant ex_type, and add an operation on ex that interprets the operator.

\section{Intricacies}

The ex type enforces that distributivity and associativity rules are always applied, but commutative semirings have more equations. In a normal form, arguments to commutative operators should be sorted according to some linear order $\prec$ : if $a \prec b$, then $a+(b+0)$ is normalised and $b+(a+0)$ is not. Defining a linear order on ex requires an order on atoms; definitional equality of atoms is tested (with user control over the amount of definitional unfolding) in the tactic monad [3], so a well-defined order on atoms cannot be easily expressed on the type level. Additionally, the recursive structure of expressions means any expression $a$ can also be represented as $(a)^{1} * 1+0$; if the left argument to ${ }^{\wedge}$ is 0 or $a * b+0$, the expression is not in normal form. Although these invariants can also be encoded in a more complicated ex type, they are instead maintained by careful programming. A mistake in maintaining these invariants is not fatal: invariants only protect completeness, not soundness, of ring_exp.

Efficient handling of numerals in expressions, using the coeff constructor, is required for acceptable running time without sacrificing completeness. The tactic should not unfold expressions like $x * 1000$ as 1000 additions of the variable $x$. Representing numerals with the coeff constructor requires an extra step to implement addition. When terms overlap, differing only in the coefficients as for $a * b^{2} * 1+a * b^{2} * 2$, their sum is given by adding their coefficients: $a * b^{2} * 3$. Moreover, when the coefficients add up to 0 , the correct representation is not $a * b^{2} * 0$ : ex prod but 0 : ex sum. Coefficients must be treated similarly in exponents: $x^{a * b^{2} * 1} * x^{a * b^{2} * 2}=x^{a * b^{2} * 3}$. Both cases are handled by a function add_overlap which returns the correct sum if there is overlap, or indicates that there is no such overlap. By choosing the order on expressions such that overlapping terms will appear adjacent in a sum, add_overlap can be applied in one linear scan.

A subtle complication arises when normalising in the exponent of an expression $\mathrm{a}^{\wedge} \mathrm{b}$ : the type of $\mathrm{a}$ is an arbitrary commutative semiring, but $\mathrm{b}$ must be $\mathrm{a}$ natural number. To correctly compute a normalised expression for $b$, the tactic needs to keep track of the type of $b$. The calculations of the eval function are thus done in an extension of the tactic monad, called the ring_exp_m monad. Using a reader monad transformer [6], ring_exp_m stores the type of the current expression as a variable which can be replaced locally when operating on exponents. 
Implementing subtraction and division also requires more work, since semirings in general do not have well-defined - or / operators. The tactic uses typeclass inference to determine whether the required extra structure exists on the type. When this is the case, the operators can be rewritten: $a-b$ becomes $a+(-1) * b$ in a ring and $a / b$ becomes $a * b^{-1}$ in a field. Otherwise, the subtraction or division is treated as an atom. Conditionally rewriting avoids the need for an almost-ring concept to treat semirings and rings uniformly [4]. Cancellation of multiplication and division, such as $a * b / a=b$, is not supported by the tactic, since such lemmas require an $a \neq 0$ side condition. In future work, extending the ex type with a negation or multiplicative inverse constructor could allow for handling of these operators in more general cases.

For completeness, atoms should be considered up to definitional equality: $(\lambda \mathrm{x}, \mathrm{x})$ a and $(\lambda \mathrm{x} y, \mathrm{x})$ a $\mathrm{b}$ reduce to the same value $\mathrm{a}$, so they should be treated as the same atom. The ring_exp_m monad contains a state monad transformer to keep track of which atoms are definitionally equal. The state consists of a list of all distinct atoms encountered in the whole input expression, and any comparisons between atoms are instead made by comparing their indices in the list. As an additional benefit, the indices induce an order on atoms, which is used to sort arguments to commutative operators. Within atoms, there may be subexpressions that can be normalised as well. Instead of running the normaliser directly, ring_exp calls the built-in tactic simp with the normaliser as an argument. The simp tactic calls a given normaliser on each subexpression, rewriting it when the normaliser succeeds.

\section{Optimisations}

An important practical consideration in implementing ring_exp is its efficiency, especially running time. Among the approximately 300 calls to ring in mathlib, about half are invocations on linear expressions by the tactic linarith. Since ring_exp is intended to work as a drop-in replacement for ring, its performance characteristics, especially for linear expressions, should be comparable.

Optimising the code was a notable part of the implementation of ring_exp. Profiling revealed that up to $90 \%$ of running time could be spent on inferring implicit arguments and typeclass instances. The solution was to pass all arguments explicitly and maintain a cache of typeclass instances, also caching the expressions for the constants 0 and 1 . It was possible to apply this solution without large changes to the codebase, because the required extra fields were hidden behind the ring_exp_m and ex_info types.

The result of these optimisations can be quantified by comparing the running time of ring and ring_exp on randomly generated expressions ${ }^{3}$. The performance measure is the tactic execution time reported by the Lean profiler, running on a $3 \mathrm{GHz}$ Intel@ Core ${ }^{\mathrm{TM}}$ i5-8500 CPU with 16 GB of RAM. On arbitrary expressions, the benchmark indicates that $r i n g \_$exp is a factor of approximately

\footnotetext{
${ }^{3}$ The benchmark program and analysis scripts are available at https://github.com/ lean-forward/ring_exp.
} 
3.9 times slower than ring; on linear expressions such as are passed by linarith, ring_exp is 1.7 times slower than ring.

Compared to a constant factor difference in the average cases, ring_exp has an advantage on problems requiring efficient handling of numeric exponents. The ring_exp tactic is a factor 20 faster than ring when showing $x^{50} * x^{50}=x^{100}$ in an arbitrary ring. A similar speedup for ring_exp was found in practice, for the goal $\left(1+x^{2}+x^{4}+x^{6}\right) *(1+x)=1+x+x^{2}+x^{3}+x^{4}+x^{5}+x^{6}+x^{7}$. The Horner normal form used by ring is optimal for representing expressions with additions and multiplications, so a constant-factor slowdown compared to ring on simpler goals is traded off for faster and more powerful handling of more complicated goals.

\section{Discussion}

The ring tactic for Coq and Lean can efficiently convert expressions in commutative semirings to normal form. A normalizing procedure for polynomials is also included with the Agda standard library [9], HOL Light [5] and Isabelle/HOL [8], and decision procedures exist that support exponential functions [1]; there is no single normalisation procedure supporting compound expressions in exponents.

Compared with the ring tactic, the ring_exp tactic can deal with a strict superset of expressions, and can do so without sacrificing too much speed. The extensible nature of the ex type should make it simple to add support for more operators to ring_exp. Independently, it should be possible to adapt the ex type to other algebraic structures such as lattices or vector spaces. Although more optimisations are needed to fully equal ring in average case efficiency, the ring_exp tactic already achieves its goal of being a useful, more general normalisation tactic. These results are as much a consequence of engineering effort as of theoretical work.

Acknowledgements. The author has received funding from the NWO under the Vidi program (project No. 016.Vidi.189.037, Lean Forward).

Floris van Doorn, Mario Carneiro and Robert Y. Lewis reviewed the code and suggested improvements. Brian Gin-Ge Chen, Gabriel Ebner, Jasmin Blanchette, Kevin Buzzard, Robert Y. Lewis, Sander Dahmen and the anonymous reviewers read this paper and gave useful suggestions. Many thanks for the help!

\section{References}

1. Akbarpour, B., Paulson, L.C.: Extending a resolution prover for inequalities on elementary functions. In: Dershowitz, N., Voronkov, A. (eds.) LPAR 2007. LNCS (LNAI), vol. 4790, pp. 47-61. Springer, Heidelberg (2007). https://doi.org/10. 1007/978-3-540-75560-9_6

2. Boutin, S.: Using reflection to build efficient and certified decision procedures. In: Abadi, M., Ito, T. (eds.) TACS 1997. LNCS, vol. 1281, pp. 515-529. Springer, Heidelberg (1997). https://doi.org/10.1007/BFb0014565 
3. Ebner, G., Ullrich, S., Roesch, J., Avigad, J., de Moura, L.: A metaprogramming framework for formal verification. In: ICFP 2017, PACMPL. ACM (2017). https:// doi.org/10.1145/3110278

4. Grégoire, B., Mahboubi, A.: Proving equalities in a commutative ring done right in Coq. In: Hurd, J., Melham, T. (eds.) TPHOLs 2005. LNCS, vol. 3603, pp. 98-113. Springer, Heidelberg (2005). https://doi.org/10.1007/11541868_7

5. Harrison, J.: HOL light: a tutorial introduction. In: Srivas, M., Camilleri, A. (eds.) FMCAD 1996. LNCS, vol. 1166, pp. 265-269. Springer, Heidelberg (1996). https:// doi.org/10.1007/BFb0031814

6. Liang, S., Hudak, P., Jones, M.: Monad transformers and modular interpreters. In: POPL 1995, pp. 333-343. ACM (1995). https://doi.org/10.1145/199448.199528

7. de Moura, L., Kong, S., Avigad, J., van Doorn, F., von Raumer, J.: The lean theorem prover (System Description). In: Felty, A.P., Middeldorp, A. (eds.) CADE 2015. LNCS (LNAI), vol. 9195, pp. 378-388. Springer, Cham (2015). https://doi. org/10.1007/978-3-319-21401-6_26

8. Nipkow, T., Wenzel, M., Paulson, L.C. (eds.): Isabelle/HOL: A Proof Assistant for Higher-Order Logic. LNCS, vol. 2283. Springer, Heidelberg (2002). https:// doi.org/10.1007/3-540-45949-9

9. The Agda Team: Agda standard library, version 1.3 for Agda 2.6.1. https://wiki. portal.chalmers.se/agda/Libraries/StandardLibrary

10. The mathlib Community: The Lean Mathematical Library. In: Blanchette, J., Hrițcu, C. (eds.) CPP 2020, pp. 367-381. ACM (2020). https://doi.org/10.1145/ 3372885.3373824 